\title{
Performance Issues with Routing in Multi-Channel Multi-Interface IEEE 802.11s Networks
}

\author{
Renato C. Juaçaba Neto ${ }^{\dagger}$, Rossana M. C. Andrade ${ }^{\dagger}$, Reinaldo B. Braga*, Fabrice Theoleyre ${ }^{\ddagger}$, Carina T. Oliveira* \\ ${ }^{\dagger}$ Federal University of Ceará (UFC), Brazil \\ *Federal Institute of Education, Science and Technology of Ceará (IFCE), Brazil

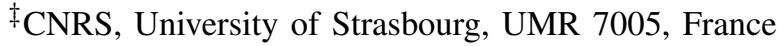 \\ Email: \{renatojuacaba\}@great.ufc.br, \{rossana\}@ufc.br, \{theoleyre\}@unistra.fr, \{reinaldo.braga,carina.oliveira $\} @ i f c e . e d u . b r$
}

\begin{abstract}
Wireless Mesh Networks have the potential to serve as an all-wireless backbone. They represent a key building block of the new Internet of Things to collect all the traffic. However, IEEE 802.11 performs very poorly in multi-hop topologies leading to unfairness and a poor throughput. Thus, the standard IEEE 802.11s proposes to re-use the IEEE 802.11 medium access in a mesh multi-hop topology while using a routing protocol to create routes at the layer 2 . IEEE 802.11s may exploit multichannel multi-interface wireless routers to increase the throughput. In particular, the routing protocol must select carefully the forwarders to limit inter- and intra-flow interference. In this paper, we propose to fully characterize the performance of IEEE 802.11s when using multi-interface nodes, and to isolate some key performance issues. We also propose some key mechanisms that must be implemented to solve these pathological cases and to improve the throughput.
\end{abstract}

Keywords-IEEE 802.11s; Multi-Interface; Routing, Performance Evaluation.

\section{INTRODUCTION}

Wireless Mesh Networks (WMN) have attracted increasing attention in recent years because of their low-cost, ease of deployment, and robustness [1]. WMN are composed of interconnected wireless routers, called mesh stations, that are dynamically self-organized, self-configured and self-healing without any centralized control [2]. In this way, they provide broadband wireless coverage to large areas without infrastructure requirements. WMN have been used in numerous application scenarios such as broadband home networking, community networks, and intelligent transportation systems.

The popularity of WMN has lead to the IEEE 802.11 standardization of a mesh networking solution for broadcast and unicast packet delivery over a multi-hop topology. The standard is called IEEE 802.11s Mesh Networking [2].

In the initial design of WMN, mesh stations were typically equipped with a single IEEE 802.11 wireless network interface card that operates over only a small portion of the available spectrum (a channel). However, it is well-known that the performance of IEEE 802.11 quickly degrades in multi-hop topologies [3]. In particular, a group of emitters may not sense the same medium activity because they are spatially far from each other. This asymmetrical view leads to unfairness. Even when we consider one single flow relayed over multiple hops (i.e., intra-flow interference), collisions impact very negatively the throughput [1].

One way of improving the performance is to use multiple orthogonal channels so that different mesh stations may simultaneously operate on different channels. For instance,
IEEE $802.11 \mathrm{a}$ provides 12 orthogonal channels in the 5 $\mathrm{GHz}$ spectrum [4]. The main goal of multi-channel singleinterface networks is to distribute the load across the available orthogonal channels. Consequently, while transmissions are multiplexed over different channels, interference and channel congestion problems are minimized [5]. However, the solution has to be carefully designed to avoid the deafness problem [4]: the emitter must know a priori the channel used by the receiver when transmitting a packet.

To fully take advantage of multiple orthogonal channels, nodes may have multiple interfaces so that different mesh stations may operate on different channels at the same time. A pair of neighboring stations may even use several interfaces to increase the throughput. In other words, full-duplex operation is possible at each node. Equipping mesh stations with multiple interfaces then leads to efficient spectrum utilization and increases the bandwidth available to nodes in the network. Such networks are often designated as Multi-Channel MultiInterface (MCMI) WMN [6].

Although there has been significant work in MCMI WMN [7], [8], [9], understanding finely the limits of IEEE 802.11 s is still an open problem. We propose here to focus on the performance issues of the Hybrid Wireless Mesh Protocol (HWMP) [2], which is the routing protocol recommended by the IEEE 802.11s standard. To the best of our knowledge, the performance evaluation of HWMP in MCMI WMN has not been addressed prior to this work. In fact, there is only few studies on the performance evaluation of HWMP in singlechannel single-interface WMN [10], [11].

In this paper, we thoroughly evaluated the behavior of IEEE 802.11s in multi-hop topologies. In particular, we highlighted the limits of HWMP to deal with multi-interface nodes: the replication of control messages leads to a misbehavior, impacting negatively the performance.

Our contribution is threefold:

- we thoroughly evaluate the behavior of IEEE 802.11s with multi-interface nodes;

- we provide a summary of the different performance issues extracted from simulations;

- we present a set of mechanisms which should be implemented to solve these performance issues.

\section{IEEE $802.11 \mathrm{~s}$}

In this section, we present the most important concepts of IEEE 802.11s [2], which is the IEEE 802.11 (Wi-Fi) 
amendment for WLAN mesh networks.

\section{A. Mesh Network Architecture}

The architecture of a WMN consists of a backbone of autonomous nodes that provides large coverage, connectivity and robustness to the network. Figure 1 gives an example of WMN, where dashed and solid lines indicate wireless and wired links, respectively.

According to the IEEE 802.11s, nodes fall into one of the following categories:

- Stations (STA) are the basic entities in an IEEE 802.11 network. They correspond to nodes that request services but do not forward frames, nor participate in path discovery mechanisms. Laptops, cell phones, smartphones, and tablets are examples of STA.

- Mesh Stations (Mesh STA) are wireless routers that implement mesh facilities such as topology construction, path selection and data forwarding. They can establish mesh peering with multiple neighbor Mesh STA, but they do not offer support to STA. They are usually stationary and, consequently, they can be permanently power-supplied and benefit from resources such as memory, energy, and computation power.

- Mesh Gates are Mesh STA with Access Point (AP) functionalities that enable the integration of the WMN with STA as well as with different wired and wireless networks like Ethernet, cellular, Wi-Fi, and sensors.

- Mesh Portals are Mesh STA integrated with gateway functions to interoperate with external networks such as the Internet.

It can be seen that IEEE 802.11s mesh networks can be incrementally deployed, one node at a time, according to the demand. Through this integrated WMN, the users of wired or wireless networks (e.g., Ethernet, cellular, Wi-Fi) can benefit from otherwise impossible services of these networks.

In this work, we focus on the issues of Mesh STA and Mesh Portal, leaving the Internet and the last hop issues (e.g., stations mobility, communication between Mesh Gates and stations, etc) to other areas of research. Moreover, we focus on IEEE 802.11s-based backbone wireless mesh networks.

\section{B. Hybrid Wireless Mesh Protocol (HWMP)}

HWMP is the path selection protocol recommended by IEEE 802.11s [12]. In particular, it uses MAC addresses to route the packets based on an IEEE 802.11 link metric. It combines the flexibility of a reactive path selection with a proactive tree-based approach. These two modes of operation provide different levels of functionalities.

The HWMP On-demand Mode allows mesh stations to communicate with each other using peer-to-peer paths (i.e. Any2Any traffic). This mode is based upon the Ad hoc OnDemand Distance Vector (AODV) protocol [13]. Specifically, a source node that does not have a valid path to the destination can use the Path REQuest (PREQ) broadcasting mechanism to discover link metric information to destination. Upon receiving a PREQ, the destination sends a unicast Path REPly (PREP) message back to the source. Intermediate nodes which have forwarded PREQs and PREPs messages create or update their own routing table to relay data packets between the source and the destination.

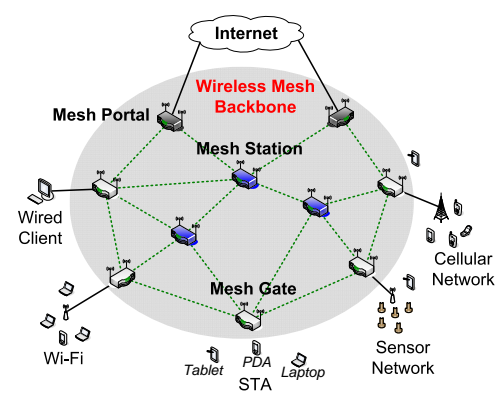

Fig. 1. IEEE 802.11s Mesh Network Architecture.

The HWMP Tree-based Proactive Mode maintains a tree path from the Mesh Portal to all other nodes. Indeed, forwarding the packets to the Mesh Portal is common when interconnecting the mesh network to the Internet. The IEEE 802.11s standard defines two mechanisms for proactively disseminating path selection information for reaching the Mesh Portal.

In the first mechanism, the root announces its presence by broadcasting a proactive PREQ. Each receiving Mesh STA updates the PREQ (TTL and path metric) and rebroadcasts it to its neighbors. As the proactive PREQs are disseminated in the entire network, a Mesh STA may receive multiple copies of a proactive PREQ. Thus, a Mesh STA updates its current path to the Mesh Portal if and only if: (i) the new PREQ contains a greater HWMP sequence number, or (ii) the HWMP sequence number is the same as the current path, but the new PREQ offers a better metric than the current path. If the proactive PREQ is sent with the Proactive PREP subfield set to 0 (respectively 1), every Mesh STA may (respectively must) respond to the received PREQ by sending a PREP back to the Mesh Portal. The overhead may consequently be significant.

As an alternative, the root may flood a Root ANNouncement (RANN). Only the Mesh STA that aims at constructing a route may reply with a PREQ forwarded to the neighbor from which the RANN is received. The root then reply with a PREP to create explicitly the route. This mode is particularly relevant when only a small subset of the Mesh STA may establish a route with the Mesh Portal.

Path ERRor (PERR) messages can be used by any of the available HWMP modes for announcing one or more unreachable destinations. The announcement is sent to all traffic sources that have an active path to the destination(s). Finally, the PERR messages reach the corresponding sources, which initiate a new path discovery cycle in order to find an alternative path.

In this work, we focus on the HWMP On-demand Mode and the first mechanism of HWMP Tree-based Proactive Mode. We have chosen this proactive mode because we evaluate the impact of HWMP in scenarios where different number of Mesh STA may establish a path with the Mesh Portal. We highlight that on-demand and tree-based proactive modes are not exclusive. In fact, they are used concurrently, since the proactive mode is an extension of the on-demand mode.

In this context, we show that HWMP is unsuitable for MCMI WMN because it does not take into consideration the high overhead incurred by the replication of control messages on multiple interfaces. Specifically, when multiple interfaces 
are used, control messages such as PREQ and PREP are retransmitted by every interface. As a result, the amount of transmitted control messages increases with the number of interfaces, which causes severe performance degradation due to heavy contention and collisions. We discuss the impact of this misbehavior of the HWMP in Section IV and present possible solutions in Section V.

\section{Airtime Link Metric (ALM)}

ALM is the default link metric of IEEE 802.11s, which is computed for each pairwise link within the mesh network. The path selection protocol accumulates all the link metric values included in the selected multi-hop path to obtain the overall cost of the path. This metric estimates the amount of channel bandwidth consumed when transmitting a frame over a link $l$. It is defined in terms of overhead, data rate, and transmission errors: $A L M_{l}=\left(O_{c a}+O_{p}+\frac{S}{r}\right) * \frac{1}{1-e_{f}}$, where $O_{c a}$ is the channel access overhead, $O_{p}$ is the protocol overhead, and $S$ is the number of bits in the test frame. The parameter $r$ is the data rate in megabits per second. The frame error rate $e_{f}$ is the probability that when a frame of standard size $S$ is transmitted at the current transmission bit rate $r$, the frame is corrupted due to transmission error. A closer look at the ALM reveals that this metric is similar to the well-know Expected Transmission Time (ETT) metric [7].

\section{Channel and Interface Assignment}

Although the importance of the channel and interface assignment in WMN [9], the current IEEE 802.11s mesh standard lets this part unspecified. The previous drafts advocated the use of a common assignment to preserve connectivity [14]. In this way, the same channel is used for all $i^{t h}$ interfaces.

Fig. 2 shows an example of the common channel assignment. Each node has two interfaces. Four orthogonal channels are available. Bold lines represent links. The channel assigned to each interface is shown inside the square brackets nearby the nodes. Note that any two neighbors have always multiple independent links to communicate with each other, resulting in a stable network topology without partitions.

\section{Simulation SetuP}

We have evaluated HWMP in MCMI WMN through extensive simulations in NS-3.19. This simulator was chosen because it already contains protocols and components of IEEE 802.11s implemented as a module of its own. We have developed extensions to detail the HWMP information captured by each interface. Besides, new features were developed to restrict simulation to fully connected scenarios (i.e., there is always a path between any two nodes). The simulation characteristics are summarized in Table I.

We have performed the evaluation in different disk scenarios with 1 Mesh Portal and 49 static Mesh STA. To represent a typical WMN traffic pattern, several flows are originated from different Mesh STA (transmitters) to the Mesh Portal. Each node has on average six neighbors in the disk scenario. Mesh STA are equipped with IEEE 802.11a wireless interfaces and therefore 12 orthogonal channels are available. On each node, interface $i$ is assigned to the orthogonal channel $C_{i}$.

Each data point in the graphical results is computed as being a result of 64 different simulations. For each simulation

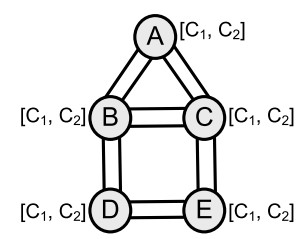

Fig. 2. Example of Common Channel Assignment in MCMI WMN.

\begin{tabular}{|l|l|}
\hline Parameter & Default Value \\
\hline Simulation Time & $80 \mathrm{~s}$ \\
\hline Number of Runs & 64 for each data point \\
\hline Network Size & 50 nodes-1 gateway \\
\hline HWMP Routing & On-demand Mode and Tree-based Proactive Mode \\
\hline Assignment Strategy & Common channel assignment \\
\hline Topology Type & Disk $($ radius $=300)$ \\
\hline Traffic Type & Poisson \\
\hline Traffic Load & 500 packets per second $(\mathrm{pps})$ \\
\hline Number of Flows & 6 \\
\hline Packet Size (bytes) & 1024 \\
\hline PHY specification & IEEE $802.11 \mathrm{a}$ \\
\hline
\end{tabular}

TABLE I. Simulation PARAMETERS.

run, a node is randomly designated as the Mesh Portal to receive the Poisson traffic generated by the transmitters. The network traffic is equally distributed among the flows.

The variations in the obtained results mainly occur due to (1) the randomness of the topology, (2) the gateway and transmitters positions, and (3) the flows components. An initial period of 10 seconds of the simulation is undertaken before the flows start ("warm-up period"). We have considered a total simulation time of 80 seconds and a packet size of 1024 bytes.

\section{RESUlts}

In this section, we present the results of the performance evaluation of HWMP. We focus on the most interesting results that show the misbehavior of HWMP in MCMI WMN. We present the results with confidence intervals of $95 \%$.

\section{A. Impact of Traffic Load}

We first investigate the impact of the traffic load on the performance of HWMP, as shown in Fig. 3. The objective is to understand the behavior of the routing protocol under different number of interfaces and network traffic.

As illustrated in Fig. 3(a), 3(b) and 3(c), in scenarios where Mesh STA have a larger number of interfaces the network has a better performance in terms of delivery rate, aggregate throughput and average end-to-end delay, respectively. This behavior is expected as the increase of interfaces also increases the number of paths using different channels. Consequently, inter-flow and intra-flow interference can be reduced. Also, note that the increase of the traffic load tends to reduce the gap between the curves in terms of delivery rate and delay. In part, this is because Mesh STA are more susceptible to suffer from quickly buffer overflow with the increase of the traffic load, especially when they are close to the Mesh Portal, which creates an unfair situation for the traffic originated by the Mesh STA close to the Mesh Portal. In this specific case, we are convinced that a solution to address this issue is to give channel and interface assignment priority to links closer to the Mesh Portal based on the number of available channels and interfaces per node (see Section V-4). 
When multiple interfaces are used, HWMP broadcasts many control messages such as PREQ (Fig.3(d)) and PREP (Fig.3(e)) on each of its interfaces. As a result, the amount of transmitted control messages increases with the number of interfaces, which causes severe performance degradation due to heavy contention and collisions. We refer to these problems associated with flooding as the broadcast storm problem [9]. As a result, a considerable part of transmission opportunities is used to send control packets instead of data packets.

Besides, Fig. 3(d) presents a clear dominance of PREQ over the other HWMP control packets: PREP (Fig. 3(e)) and PERR (Fig. 3(f)). Another important issue seen on all scenarios is the dominance of PREQ forwarded (Fig. 3(g)) over initiated PREQ (Fig. 3(h)) and initiated proactive PREQ (Fig. 3(i)). Similarly, the number of PREP forwarded (Fig. 3(j)) is superior to the number of initiated PREP (Fig. 3(k)).

\section{B. Impact of the Number of Flows}

Next, we investigate the impact of the number of flows (cf. Fig. 4). The objective is to evaluate the behavior of the MCMI WMN when the traffic is distributed through the network.

Similarly to the previous results, we observe that networks composed of Mesh STA with more interfaces perform better concerning the delivery rate (Fig. 4(a)), the aggregate throughput (Fig. 4(b)) and the end-to-end delay (Fig. 4(c)). Also, note that the delay slightly increases with the number of flows whatever the number of interfaces is. The main reason is that HWMP does not perform load-balancing and then does not seek to distribute the various flows through different paths in order to prevent channel congestions (i.e., bottleneck links). More specifically, HWMP tends to forward new flows through already established paths, which means that all flows are led to the same congested areas. Consequently, it results in poor quality paths that spend more time to transmit data because of the high level of interference and contention. In this context, we consider that HWMP performance can be improved if dynamic characteristics of the network are considered to perform routing, such as the link quality and the traffic load (see Sections V-1 and V-3).

Figures 4(d), 4(e) and 4(f) show that the amount of transmitted control messages increases considerably with the number of flows. As the previous results, the number of transmitted PREQ is superior to other HWMP control packets (PREP and PERR). Besides, it is clear the dominance of PREQ forwarded (Fig. 4(g)) over initiated PREQ (Fig. 4(h)) and initiated proactive PREQ (Fig. 4(i)).

\section{Possible Solutions}

The problems highlighted in the previous section are mainly due to the exploitation of multiple interfaces. HWMP was not designed originally to create a route through different interfaces. We present here four mechanisms that may improve the IEEE 802.11s efficiency.

1) Metrics Quantification: Many routing metrics are not able to depict all the factors that impact the performance of MCMI WMN: the exhaustive identification of all these factors is not trivial. Among the well-known factors, the inter- and intra-flow interference, the traffic load and the link quality can be regarded as the most recent trend to depict the behavioral aspects of the network. For example, interference can be measured to favor paths with channel diversity, while routing solutions aware of the traffic load may prevent congested areas. Also, link quality measures may be used to avoid links with high packet losses. However, most routing metrics do not consider the combination of these factors when judging the goodness of a path. Besides, they use different information gathering methods to obtain the measurements. In consequence, each metric has an appropriate use case, which generally is not extensible to different network scenarios (e.g., mesh networks with variations of traffic and interference).

2) Route Discovery through Multiple Interfaces: When forwarding a PREQ, a node should select the channel to use based on the channels which will be selected further in the route: channel selection is not independent among the different hops. In particular, the same channel should not be used by two interfering hops. Consequently, a node cannot filter the PREQ received through one interface just because the same PREQ has already been relayed through another interface. Thus, PREQ should be independently considered through all the interfaces.

However, multiple radio links between each pair of Mesh STA may be exploited to reduce the complexity of HWMP. We are convinced that each node should be able to piggyback in its PREQ the information of all the links with the previous hop. Else, the interdependency would create a PREQ storm: to explore all the possible combinations of channels, each node would have to forward several PREQ per interface. Since the overhead would grow with the path length, an additional mechanism should filter this information, selecting the best choice for the beginning of the path.

3) Bandwidth Estimation: Different flows may interfere with each other locally. Since IEEE 802.11 does not permit to share fairly and linearly the bandwidth among the competing flows, we should be able to estimate the available residual bandwidth.

We may exploit the mechanism described in [15] to modify the Airtime Link Metric. A model of the medium access should translate the number of retransmissions into a channel occupancy. Coupled with a call admission, we should be able to select the path minimizing the ALM while respecting the requested bandwidth for the flow.

4) Channel and Interface Assignment: A key challenge in MCMI WMN consists in achieving an effective channel and interface assignment (mapping) to decide "when to switch interfaces" as well as "which channel to assign". There is an inherent trade-off between connectivity and interference [9]. The more interfaces are assigned to the same channels, the better connectivity. However, interference and contention have oppositely a negative impact on network capacity.

In this way, we are convinced that a promising approach for improvement of HWMP consists in combining routing with assignment according to the network conditions (e.g., number of available channels and interfaces, traffic behavior, neighborhood, interference, QoS requirements, etc).

Surprisingly, to the best of our knowledge, the impact of channel and interface assignment strategies on the HWMP performance has never been studied, although it is a major component of network operation. 


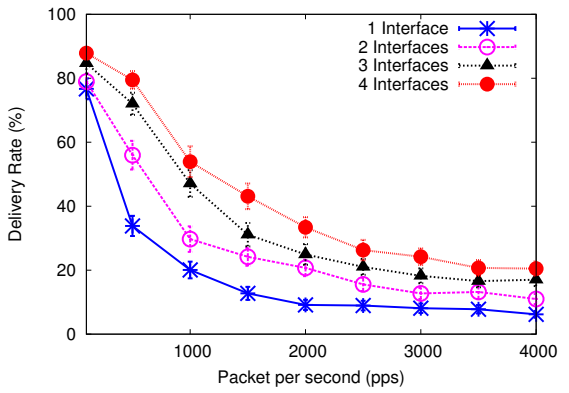

(a) Delivery Rate.

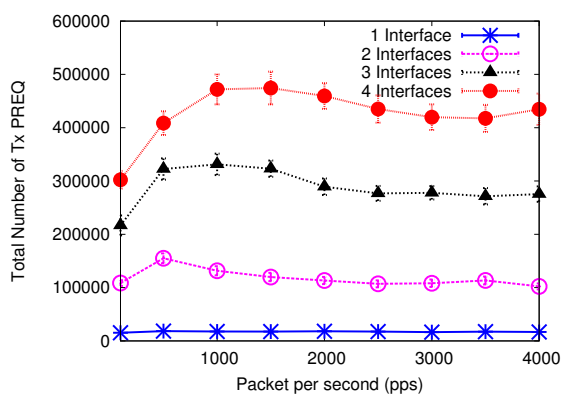

(d) Total PREQ.

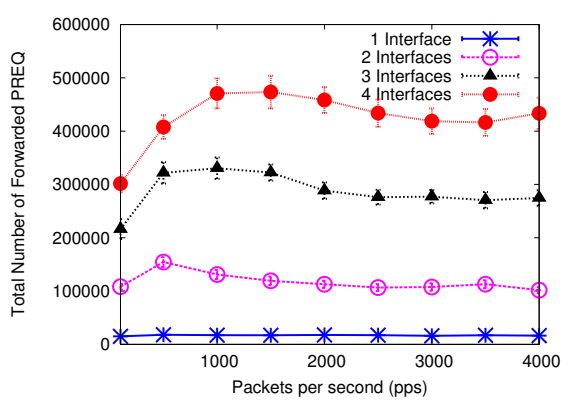

(g) Forwarded PREQ.

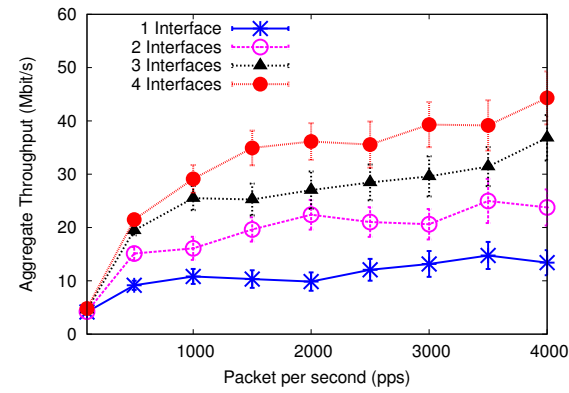

(b) Aggregate Throughput.

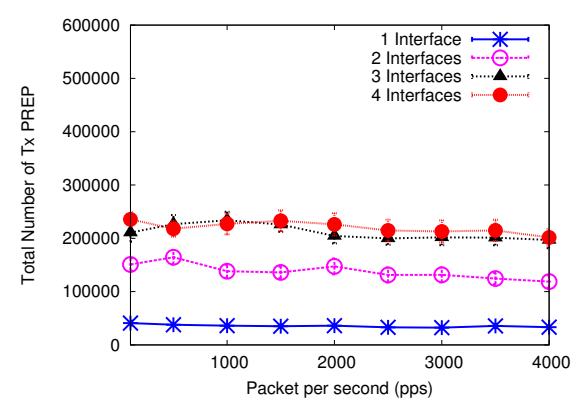

(e) Total PREP.

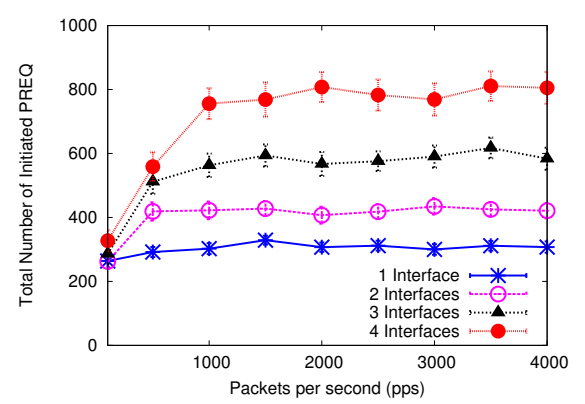

(h) Initiated PREQ.

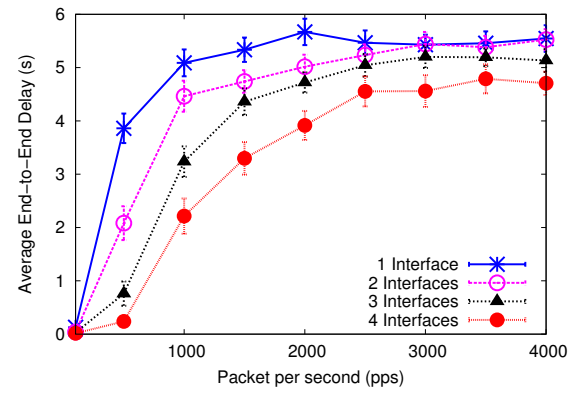

(c) Average End-to-End Delay.

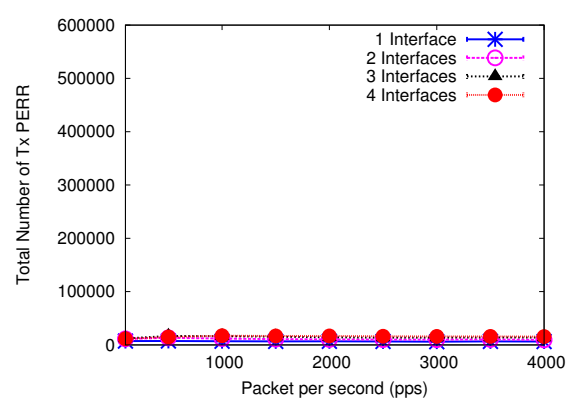

(f) Total PERR.

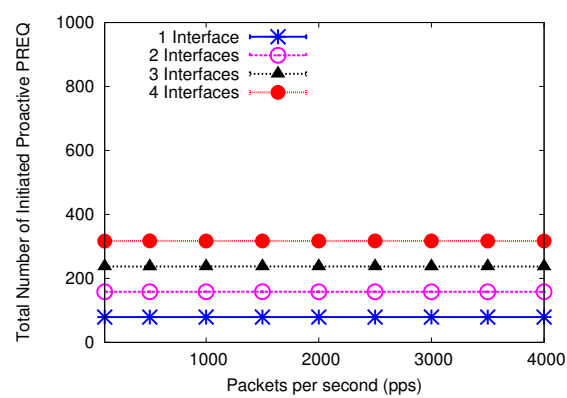

(i) Initiated Proactive PREQ.

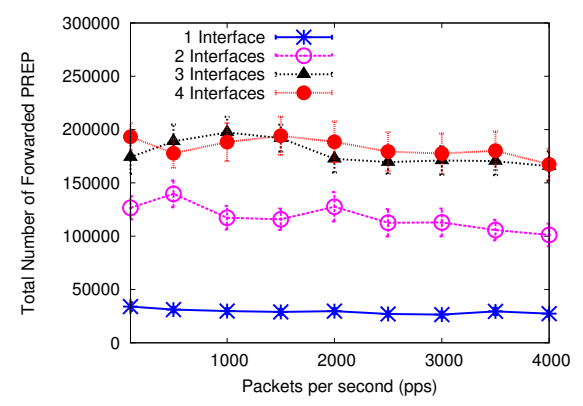

(j) Forwarded PREP.

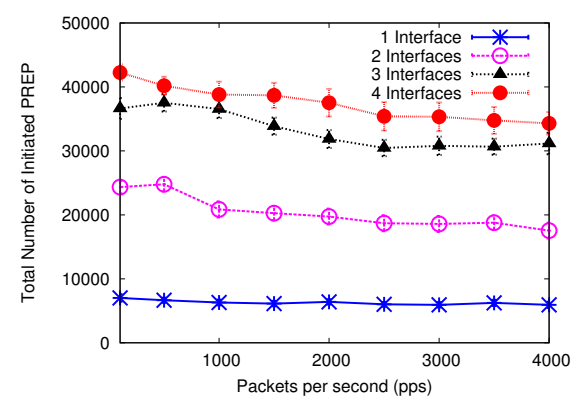

(k) Initiated PREP.

Fig. 3. Impact of Traffic Load - 50 nodes (1 Mesh Portal), 6 flows.

\section{CONClusions}

In this paper, we summarize the performance issues that arise with HWMP when used in MCMI WMN. The results and discussions provide guidelines for academic researchers, research engineers, and practitioners in planning MCMI IEEE 802.11s, as well as to improve HWMP.

The contributions presented in this paper bring up interesting perspectives for future research. For instance, we plan to evaluate the HWMP performance when different channel and interface assignment strategies are adopted. Also, we plan the development of a cross-layer routing solution for MCMI WMN with a precise characterization of the wireless links in order to increase the overall network performance.

\section{ACKNOWLEDGEMENTS}

The authors thank $\mathrm{CNPq}$, which provided financial support through the Notice MCT/CNPq 14/2013-Universal $\mathrm{n}^{\mathrm{o}} 486287 / 2013-0$ (Project Cross-Layer Optimization in MultiInterface Wireless Mesh Networks). The authors would also like to thank CENAPAD-UFC for computational facilities. 


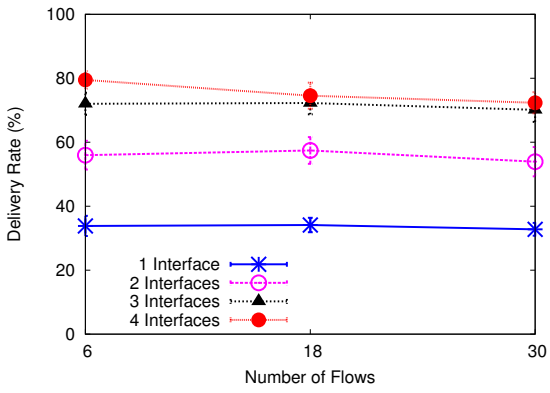

(a) Delivery Rate.

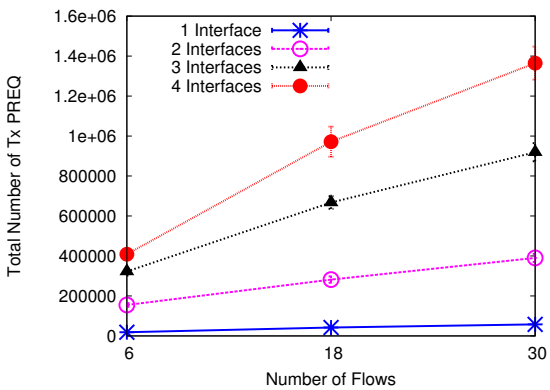

(d) Total PREQ.

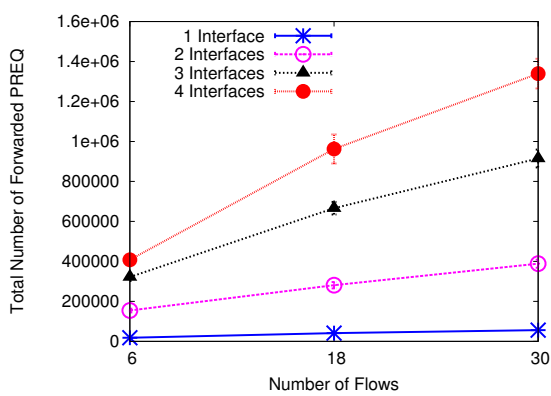

(g) Forwarded PREQ.

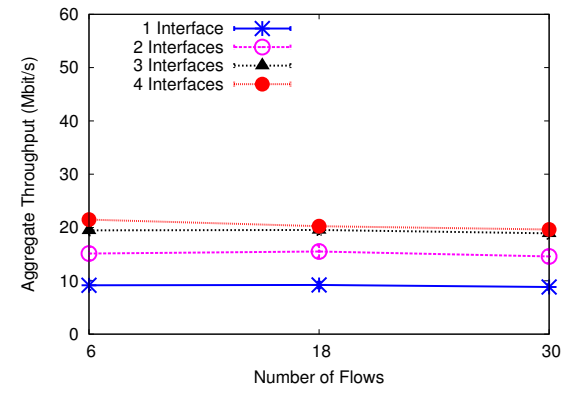

(b) Aggregate Throughput.

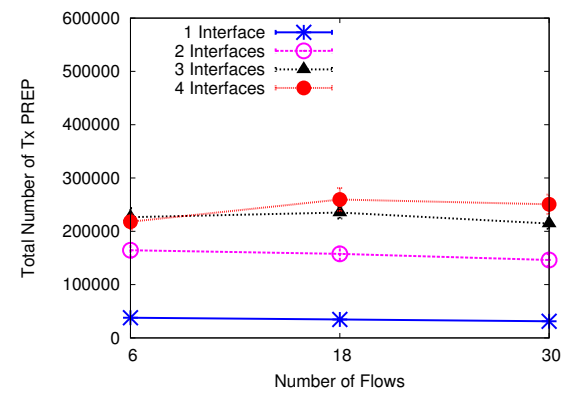

(e) Total PREP.

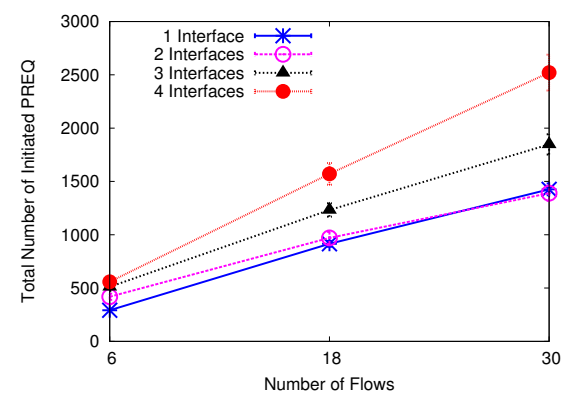

(h) Initiated PREQ.

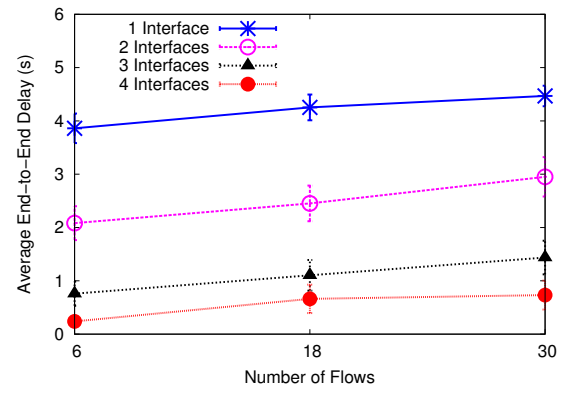

(c) Average End-to-End Delay.

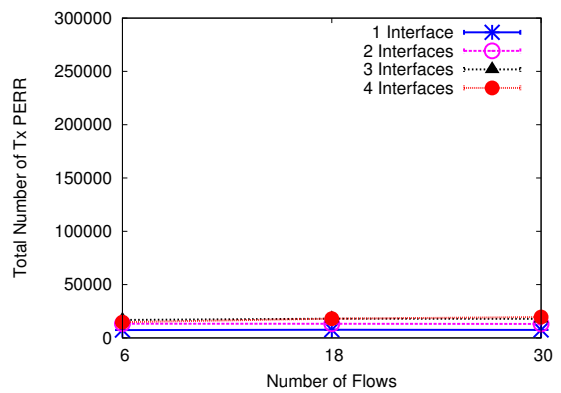

(f) Total PERR.

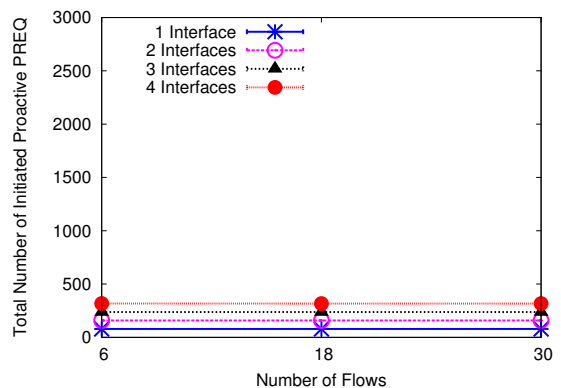

(i) Initiated Proactive PREQ.

Fig. 4. Impact of the Number of Flows - 50 nodes (1 Mesh Portal), 500 pps.

\section{REFERENCES}

[1] M. E. M. Campista, P. M. Esposito, I. M. Moraes, L. H. M. Costa O. C. M. Duarte, D. G. Passos, C. V. N. De Albuquerque, D. C. M Saade, and M. G. Rubinstein. Routing Metrics and Protocols for Wireless Mesh Networks. IEEE Network, 22(1):6-12, 2008.

[2] IEEE 802.11s. IEEE Standard for Information Technology - Telecommunications and Information Exchange Between Systems - Local and Metropolitan Area Networks - Specific Requirements - Part 11: Wireless LAN Medium Access Control (MAC) and Physical Layer (PHY) Specifications - Amendment 10: Mesh Networking, IEEE Std., 2011.

[3] C. Chaudet, D. Dhoutaut, and I. Guerin-Lassous. Performance Issues with IEEE 802.11 in Ad Hoc Networking. IEEE Communications Magazine, 43(7):110-116, 2005.

[4] J. Crichigno, M.-Y. Wu, and W. Shu. Protocols and Architectures for Channel Assignment in Wireless Mesh Metworks. Ad Hoc Networks, 6, 2008.

[5] P. Li, N. Scalabrino, Y. Fang, E. Gregori, and I. Chlamtac. How to Effectively Use Multiple Channels in Wireless Mesh Networks. IEEE Transactions on Parallel and Distributed Systems, 20(11), 2009.

[6] W. Si, S. Selvakennedy, and A. Y. Zomaya. An Overview of Channel Assignment Methods for Multi-Radio Multi-Channel Wireless Mesh Networks. Journal of Parallel and Distributed Computing, 70(5):505524, 2010.

[7] R. Draves, J. Padhye, and B. Zill. Routing in Multi-Radio, Multi-Hop Wireless Mesh Networks. In Proc. ACM MobiCom, 2004.
[8] A. A. Pirzada, M. Portmann, and J. Indulska. Performance Analysis of Multi-Radio AODV in Hybrid Wireless Mesh Networks. Computer Communications, 31(5):885-895, 2008.

[9] C. T. De Oliveira, F. Theoleyre, and A. Duda. Connectivity in MultiChannel Multi-Interface Wireless Mesh Networks. In Proc. IEEE IWCMC, 2011

[10] S. M. S. Bari, F. Anwar, and M. H. Masud. Performance study of Hybrid Wireless Mesh Protocol (HWMP) for IEEE 802.11s WLAN mesh networks. In Proc. ICCCE, 2012.

[11] M. Guesmia, M. Guezouri, and M. Mbarek. Performance Evaluation of the HWMP Proactive Tree Mode for IEEE 802.11s based Wireless Mesh Networks. In Proc. ComNet, 2012.

[12] R. C. Carrano, L. C. S. Magalhaes, D. C. M. Saade, and C. V. N. Albuquerque. IEEE 802.11s Multihop MAC: A Tutorial. IEEE Communications Surveys \& Tutorials, 13(1):52-67, 2011.

[13] C. Perkins, E. Belding-Royer, and S. Das. Ad hoc On-Demand Distance Vector (AODV) Routing, RFC 3561, IETF Network Working Group, 2003

[14] J. D. Camp and E. W. Knightly. The IEEE 802.11s Extended Service Set Mesh Networking Standard. IEEE Communications Magazine, 46(8):120-126, 2008.

[15] N. V. Nguyen, I. Guerin-Lassous, V. Moraru, and C. Sarr. Retransmission-based Available Bandwidth Estimation in IEEE 802.11based Multihop Wireless Networks. In Proc. ACM MSWiM, 2011. 